\title{
Questioning the need for ICU level of care in pediatric patients following elective uncomplicated craniotomy for brain tumors
}

\author{
Brandon C. Gabel, MD, ${ }^{1}$ Joel Martin, MD, ${ }^{1}$ John R. Crawford, MD, MS, ${ }^{2}$ and \\ Michael Levy, MD, PhD ${ }^{1,3}$
}

\begin{abstract}
1Department of Neurosurgery, University of California, San Diego; '2Department of Neurosciences and Pediatrics, University of California, San Diego, and Rady Children's Hospital; and 'Department of Neurosurgery, Rady Children's Hospital, San Diego, California
\end{abstract}

\begin{abstract}
OBJECTIVE The object of this study is to address what factors may necessitate the need for intensive care monitoring after elective uncomplicated craniotomy in pediatric patients who are initially managed in a non-intensive care unit setting postoperatively.

METHODS A retrospective chart review was undertaken for all patients who underwent elective craniotomy for brain tumor between April of 2007 and April of 2012 and who were directly admitted to the floor postoperatively. Factors such as age, tumor type, craniotomy location, neurological comorbidities, reason for transfer to intensive care unit (ICU) level of care (if applicable), time between admittance to floor and transfer to ICU level of care, and reason for transfer to ICU level of care were assessed.
\end{abstract}

RESULTS Adjusted logistic regression found 2 significant positive predictors of postoperative transfer to the ICU after initial admission to the floor: primitive neuroectodermal tumor pathology (OR 44.10,95\% Cl 1.24-1572.16, $p=0.04)$, and repeat craniotomy during the same hospitalization (OR 13.97, 95\% $\mathrm{Cl} 1.21-160.66, p=0.03)$. Conversely, 1 negative factor was found: low-grade glioma pathology (OR 0.05, 95\% Cl 0.00-0.87, p = 0.04).

CONCLUSIONS Select pediatric patients may not require ICU level of care after elective uncomplicated pediatric craniotomy. Additional studies are needed to adequately address which patients would benefit from initial ICU admittance following elective craniotomies for brain tumors.

http://thejns.org/doi/abs/10.3171/2015.8.PEDS15191

KEY WORDS brain tumor; intensive care unit; craniotomy; oncology

$\mathrm{M}$ ANY neurosurgeons admit patients with elective uncomplicated craniotomies to an intensive care unit (ICU) for postoperative monitoring. Numerous studies in adults have shown that these patients can be safely monitored in a step-down unit or on the surgical floor. ${ }^{1,4,8}$ Therefore, the practice of admitting to an ICU postoperatively has come under scrutiny as costly and potentially unnecessary.

The cost of intensive care, both financially and logistically, is substantial. One study showed that intensive care, although representing only a small proportion of total care, accounted for a substantial portion of total cost. ${ }^{6}$ Countries with socialized health care have been concerned over the cost of routine post-ICU admission, especially when the benefits remain questionable. ${ }^{8,9,12}$

Most studies addressing the need for intensive care af- ter elective craniotomy include only adult patients. Some institutions have shown low complication rates in patients who underwent outpatient craniotomy. ${ }^{2,3,5}$ However, it is important to realize that adult patients may have medical comorbidities that require intensive care independent of their neurological disease?

Admittance to an ICU has been associated with negative psychiatric sequelae. For example, Ratzer et al. illustrated that in trauma patients nearly $20 \%$ reported high levels of posttraumatic stress disorder symptoms at 6-months follow-up..$^{10}$ Interestingly, and perhaps more salient to a pediatric population, a recent study from Australia showed that anxiety and posttraumatic stress disorder symptoms were common in next of kin relatives of patients admitted to an ICU. ${ }^{11}$

The practice of admitting pediatric patients to the ICU 
postcraniotomy is also commonplace. Pediatric patients uncommonly have the coexistent medical problems that adults have, but admittance to the ICU remains standard practice in most institutions. On the other hand, some pediatric patients with complex genetic syndromes can be medically complicated, which may warrant ICU admission after craniotomy. Regardless, most admissions to the ICU are likely related to the fear of delayed postoperative surgical complications that may be missed in a non-ICU setting.

In our institution, most elective craniotomies for brain tumors without intraoperative complications are sent to the floor postoperatively, assuming the patient is extubated in the postanesthesia care unit and does not have new neurological deficits. The aim of this study is to address the frequency and reasons why transfer to an ICU level of care, after elective admission to the floor, may be necessary.

\section{Methods \\ Study Population}

Following approval by the University of California, San Diego, institutional review board and Rady Children's Hospital, data on pediatric patients undergoing elective craniotomy for a diagnosis of brain tumor between April of 2007 and April of 2012 were retrospectively collected. The following International Classification of Diseases, Ninth Revision (ICD-9) codes were used to query the database: 01.59 (other excision or destruction of lesion or tissue of brain), 01.24 (other craniotomy), 01.23 (reopening of craniotomy site), 07.53 (excision of pineal body), and 01.14 (open biopsy of brain).

The primary aim of this study was to assess risk factors for postoperative transfer from a non-ICU level of care to an ICU level of care after craniotomy for tumor resection in pediatric patients. Patient records and information were de-identified prior to analysis. For this retrospective study, 61 patients who underwent craniotomy for tumor at our institution during the study period met the inclusion criteria. The inclusion criteria included: 1) age less than 18 years at the time of admission of those who underwent elective craniotomy surgery for tumor; 2) patients admitted directly to a non-ICU level of care postoperatively; and 3 ) patients with documented variables in hospital charts. Exclusion criteria included patients who presented to the emergency department, patients transferred from another facility, or patients requiring an external ventricular or lumbar drain postprocedure. Patients who had evidence of known intraoperative complications, patients who were not extubated postoperatively, and patients with postoperative neurological deficits were sent to the pediatric ICU postoperatively; these patients were also excluded from analysis. Additionally, neither the age of the patient nor the length of surgery was a contributing factor to what level of care the patient was admitted to postoperatively.

\section{Data Collection}

Clinical and operative variables were collected for each patient, including age, sex, comorbidities (epilepsy, hydrocephalus, autism, attention-deficit disorders [ADDs], neurofibromatosis, tuberous sclerosis, and aplastic anemia), tumor pathology, type of craniotomy (supratentorial or infratentorial), length of stay, and number of days at each level of care. The variable infratentorial represents a combination of the variables infratentorial intraventricular, infratentorial extraventricular, and cerebellopontine angle tumor. The variable supratentorial represents a combination of the variables supratentorial intraventricular, supratentorial intraventricular midline, supratentorial extraventricular eloquent, and supratentorial extraventricular noneloquent.

Additionally, immediate complications, defined as those occurring during the hospital admission, were noted. The reason for transfer to the ICU, if needed, was also obtained from progress notes. If applicable, the amount of time between admission to a non-ICU level of care and transfer to the ICU was also noted. In variables with an adequate cohort, associations between postoperative ICU transfer and individual risk factors in all patients were determined using logistic regression analysis that was adjusted for preoperative demographic variables. Significance of the results was assessed at $\alpha=0.05$. Analysis was conducted using $\mathrm{R}$ language.

\section{Results}

Using the ICD-9 procedure codes listed above, 177 patients were identified. Of these 177 patients, 61 met inclusion criteria. The remaining patients were excluded because they were admitted directly to the ICU either pre- or postsurgery, were transferred from another facility, or presented to the emergency department. There were 34 males $(56 \%)$ and 27 females (44\%). The average age at admission was $8.3 \pm 4.7$ years, and the average length of follow-up in the neurosurgery clinic was 1.82 years (range 10 days to 5.82 years; Table 1$)$.

When comorbidities were analyzed, 13 patients (21\%) had a history of epilepsy, 9 (15\%) had hydrocephalus, 3 (4.9\%) had autism, 3 (4.9\%) had attention-deficit hyperactivity disorder (ADHD)/ADD, 3 (4.9\%) had tuberous sclerosis, 2 (3.3\%) had neurofibromatosis Type 1 (NF1) or 2 (NF2), and $1(1.6 \%)$ had aplastic anemia (Table 1).

In terms of pathology, 36 patients (59\%) had low-grade gliomas (defined as pilocytic astrocytoma, oligodendroglioma, subependymal giant cell astrocytoma, schwannoma, low-grade ependymoma, xanthoastrocytoma, choroid plexus papilloma, ganglioglioma), 6 (9.8\%) had highgrade gliomas (defined as glioblastoma, other high grade tumor not otherwise specified, high grade ependymoma, and choroid plexus carcinoma), 6 (9.8\%) had primitive neuroectodermal tumors (PNETs), which included medulloblastomas, 3 (4.9\%) had intracranial epidermoid/dermoids, 2 (3.3\%) had dysembyroplastic neuroepithelial tumors (DNETs), 1 (1.6\%) had an atypical teratoid/rhabdoid tumor, $1(1.6 \%)$ had a craniopharyngioma, $1(1.6 \%)$ had a fibrosarcoma, $1(1.6 \%)$ had an osteosarcoma, $1(1.6 \%)$ had a yolk sac tumor, $1(1.6 \%)$ had a meningoepithelial tumor, $1(1.6 \%)$ had meningoangiomatosis, and $1(1.6 \%)$ had a malignant spindle cell tumor (Table 1).

Thirty-three patients (54\%) underwent supratentorial surgery and $28(45.9 \%)$ had infratentorial surgery. Twelve patients $(20 \%)$ had repeat craniotomies, with 5 (8.2\%) undergoing the repeat craniotomy during the same hospitalization, versus 8 (13.1\%) having a craniotomy during 
TABLE 1. Adjusted risk factor assessment for postoperative transfer from the hospital floor to the ICU in pediatric craniotomy for tumor resections

\begin{tabular}{|c|c|c|c|c|}
\hline Variable & $\begin{array}{l}\text { Not Transferred to ICU } \\
\qquad(n=55)^{*}\end{array}$ & $\begin{array}{l}\text { Transferred to ICU } \\
\qquad(n=6)^{*}\end{array}$ & OR $(95 \% \mathrm{Cl})$ & $\mathrm{p}$ Value \\
\hline \multicolumn{5}{|l|}{ Preop/demographic variables } \\
\hline Mean age in yrs (SD) & $8.2(4.6)$ & $9.0(5.9)$ & $1.03(0.87-1.23)$ & 0.71 \\
\hline Males & $30(54.5)$ & $4(66.7)$ & $2.31(0.37-14.43)$ & 0.37 \\
\hline Epilepsy & $12(21.8)$ & $1(16.7)$ & $0.00(0.00-9.44)$ & 0.12 \\
\hline Hydrocephalus & $8(12.7)$ & $1(16.7)$ & $1.28(0.12-13.34)$ & 0.83 \\
\hline Autism & $3(5.5)$ & $0(0.0)$ & - & - \\
\hline ADHD/ADD & $3(3.6)$ & $0(0.0)$ & - & - \\
\hline NF1 & $2(3.6)$ & $0(0.0)$ & - & - \\
\hline Tuberous sclerosis & $2(3.6)$ & $1(16.7)$ & - & - \\
\hline Aplastic anemia & $1(1.8)$ & $0(0.0)$ & - & - \\
\hline \multicolumn{5}{|l|}{ Pathology } \\
\hline Low-grade glioma & $35(63.6)$ & $1(16.7)$ & $0.05(0.00-0.87)$ & $0.04 \dagger$ \\
\hline High-grade glioma & $5(9.1)$ & $1(16.7)$ & $1.17(0.09-15.86)$ & 0.91 \\
\hline PNET & $4(7.3)$ & $2(33.3)$ & $44.10(1.24-1572.16)$ & $0.04 \dagger$ \\
\hline Epidermoid/dermoid & $3(5.5)$ & $0(0.0)$ & - & - \\
\hline Atypical teratoid/rhabdoid tumor & $1(1.8)$ & $0(0.0)$ & - & - \\
\hline Craniopharyngioma & $0(0.0)$ & $1(16.7)$ & - & - \\
\hline Osteosarcoma & $0(0.0)$ & $1(16.7)$ & - & - \\
\hline DNET & $2(3.6)$ & $0(0.0)$ & - & - \\
\hline Fibrosarcoma & $1(1.8)$ & $0(0.0)$ & - & - \\
\hline Meningoepithelial tumor & $1(1.8)$ & $0(0.0)$ & - & - \\
\hline Meningoangiomatosis & $1(1.8)$ & $0(0.0)$ & - & - \\
\hline Malignant spindle cell tumor & $1(1.8)$ & $0(0.0)$ & - & - \\
\hline Yolk sac tumor & $1(1.8)$ & $0(0.0)$ & - & - \\
\hline \multicolumn{5}{|l|}{ Craniotomy } \\
\hline Supratentorial & $29(50.9)$ & $4(66.7)$ & $2.24(0.27-18.80)$ & 0.46 \\
\hline Infratentorial & $26(47.3)$ & $2(33.3)$ & $0.48(0.06-3.95)$ & 0.49 \\
\hline Repeat craniotomy & $9(16.4)$ & $3(50.0)$ & $6.02(0.84-43.35)$ & 0.07 \\
\hline Repeat craniotomy (same hospital stay) & $3(5.5)$ & $2(33.3)$ & $13.97(1.21-160.66)$ & $0.03 \dagger$ \\
\hline Repeat craniotomy (prior hospitalization) & $7(12.7)$ & $1(16.7)$ & $1.14(0.09-15.16)$ & 0.92 \\
\hline
\end{tabular}

a prior hospitalization. Three patients (4.9\%) underwent repeat craniotomy during the same hospital stay for residual disease, and 2 (3.3\%) underwent repeat craniotomy for postoperative hematoma (Table 1).

The average length of stay was $5.3 \pm 8.0$ days. Six patients (9.8\% of all patients) were transferred to the ICU after having previously been admitted to the floor. For these patients, the average time spent in the ICU was 8.83 days. The reasons for transfer to the ICU were varied. The first patient was transferred on postoperative Day 2 for respiratory distress manifesting as an increasing oxygen requirement on the floor, although this patient never required intubation. The second patient suffered invasive osteosarcoma of the skull, brain, and sagittal sinus, and was transferred to the ICU on postoperative Day 2 for status epilepticus. A repeat head CT scan performed at the time of transfer demonstrated a recurrent intracranial hemorrhage requiring emergency repeat craniotomy; this patient ultimately died as a result of multiple cerebral infarctions. The third patient had a known history of tuberous sclerosis and epilepsy and was transferred on postoperative Day 1 for increasing seizure frequency above baseline. The seizures were managed effectively in the ICU with a change in antiepileptic medication. The fourth patient was transferred for decreased mental status on postoperative Day 0; a repeat head CT scan showed intracranial hemorrhage that was managed with emergency reoperation and ligation of a bleeding vessel. The fifth patient, who underwent resection of a craniopharyngioma, was transferred on postoperative Day 4 for hyponatremia consistent with cerebral salt wasting; this was managed in the ICU with a $3 \%$ saline infusion. The sixth patient was transferred on postoperative Day 2 for bradycardia that required no specific intervention and resolved on its own in the ICU. There was 1 death (1.6\% of all patients) during the same hospitalization as the elective craniotomy (Table 2). 
TABLE 2. Reason for transfer to the ICU setting after admission to the surgical floor

\begin{tabular}{cllccc}
\hline $\begin{array}{c}\text { Case } \\
\text { No. }\end{array}$ & \multicolumn{1}{c}{ Tumor Type } & \multicolumn{1}{c}{ Reason for Transfer } & $\begin{array}{c}\text { Timing of Transfer } \\
\text { (postop day) }\end{array}$ & $\begin{array}{c}\text { Days } \\
\text { in ICU }\end{array}$ & Outcome \\
\hline 1 & Medulloblastoma & Mild respiratory distress & 2 & 3 & Neurologically intact at follow up \\
\hline 2 & Osteosarcoma & $\begin{array}{c}\text { Status epilepticus; intracranial hemor- } \\
\text { rhage }\end{array}$ & 2 & 5 & Death \\
\hline 3 & $\begin{array}{c}\text { Subependymal giant cell } \\
\text { astrocytoma }\end{array}$ & $\begin{array}{c}\text { Increased seizure frequency from } \\
\text { baseline }\end{array}$ & 1 & 3 & Neurologically intact at follow-up \\
\hline 4 & $\begin{array}{c}\text { High-grade thalamic glioma } \\
\text { Altered mental status secondary to } \\
\text { hemorrhage in surgical bed }\end{array}$ & 0 & 37 & Lost to long-term follow-up \\
\hline 5 & Craniopharyngioma & $\begin{array}{c}\text { Cerebral salt wasting requiring 3\% } \\
\text { infusion }\end{array}$ & 4 & 2 & $\begin{array}{c}\text { Panhypopituitarism, bitemporal hemianop- } \\
\text { sia (present before surgery) }\end{array}$ \\
\hline 6 & Medulloblastoma & Bradycardia & 2 & 3 & Neurologically intact at follow-up \\
\hline
\end{tabular}

Adjusted logistic regression found 2 significant positive predictors of postoperative transfer to the ICU: PNET pathology (OR 44.10, 95\% CI 95\% 1.24-1572.16, $\mathrm{p}=0.04$ ), and repeat craniotomy during the same hospitalization (OR 13.97, 95\% CI 1.21-160.66, $\mathrm{p}=0.03$ ). Conversely, 1 negative factor was found: low-grade glioma pathology (OR 0.05, 95\% CI 0.00-0.87, p = 0.04; Table 1).

\section{Discussion}

To our knowledge, this is the first paper addressing the need for ICU care after elective craniotomy for brain tumors in children. We have shown a 9.8\% (6 of 61) transfer rate back to the ICU after initially being admitted to a lower level of care. Of these 6 patients, 1 died during the admission secondary to complications from intracranial hemorrhage. The remaining patients who were transferred were treated effectively for their underlying condition (1 for respiratory distress, 1 for bradycardia, 1 for cerebral salt wasting, 1 for status epilepticus, and 1 for increasing seizure frequency) and were eventually discharged from the ICU in stable condition.

Only 2 variables met statistical significance for transfer to the ICU after elective admission to a lower level of care. Patients who underwent repeat craniotomy during the same hospital stay were at increased risk. Of the 6 patients who were transferred to the ICU, 3 of them had 2 craniotomies during their hospital stay. Two of these were for postoperative hematoma, and 1 was for residual disease detected on repeat MRI. One of these patients had poorly controlled osteosarcoma that had invaded into the sinus and brain despite maximal medical and prior surgical therapy. He died after sustaining an intracranial hematoma that manifested as status epilepticus on postoperative Day 2. Given the aggressiveness of the patient's tumor despite previous resections and failed medical therapy, it is likely this patient would have succumbed to his disease in the near future. Overall, we are not surprised by this data, as one of the most common reasons for admitting patients to an ICU setting postcraniotomy is to monitor for neurological deterioration secondary to hematoma formation in the surgical bed. The third patient who underwent repeat craniotomy for residual disease had asymptomatic bradycardia of unclear significance; he was transferred for cardiovascular monitoring, and it could be argued that this may have been unnecessary.
PNET (including medulloblastoma) pathology was also associated with a statistically significant risk of transfer to an ICU level of care. All of the PNETs in our series were located in the posterior fossa. The 2 patients with PNETs who were transferred to the ICU both had signs or symptoms related to neuroanatomy of the posterior fossa: 1 patient had transient bradycardia and the other transient respiratory distress. Both of these symptoms abated without specific intervention while the patient was being monitored in the ICU. Given the proximity of the brainstem and cranial nerves to posterior fossa lesions, it is not surprising that this tumor type, which is usually large, aggressive, and frequently encases cranial nerves and brainstem structures, was associated with transfer to the ICU. Interestingly, infratentorial craniotomy as a variable was not, by itself, associated with transfer to the ICU. This is likely because less aggressive and less-invasive tumors such as pilocytic astrocytomas were represented in this cohort of patients.

Low-grade gliomas were negatively associated with transfer to the ICU. This is not a surprising finding given the nonaggressive nature of these lesions in general. Only 1 of 6 patients transferred had a low-grade glioma (subependymal giant cell astrocytoma). This patient also had tuberous sclerosis and a history of epilepsy. She was transferred back to the ICU for an increase in her baseline seizure frequency. It is not apparent that she was ever in status epilepticus, and her seizure frequency was reduced back to baseline with titration of her antiepileptic medications.

Patients with hypothalamic and pituitary dysfunction may have significant metabolic and hormonal changes that require intensive care. Monitoring patients with tumors involving the hypothalamus or pituitary gland postoperatively in an ICU setting is likely prudent given our experience. One patient with craniopharyngioma initially did well on the floor, but was transferred back to the ICU for cerebral salt wasting requiring hypertonic saline solutions several days postoperatively. The patient's sodium level eventually normalized. We were unable to show associated statistical data on this tumor type because only 1 patient was represented. We believe that tumors involving the hypothalamic and/or pituitary axis should be managed expectantly in an ICU setting given hormonal, fluid, and electrolyte disturbances that are common in these patients.

At our institution, the pediatric surgical floor is a nonspecialty specific floor where postsurgical patients are 
managed. Nurses have training in managing surgical patients, but are not specially trained to deal with neurosurgical patients. Basic neurological examinations can be performed every 4 hours at a maximum, and the floor has telemetry capabilities. The nursing to patient ratio ranges from 1:2 to 1:4. Each patient has a private room with fold-out beds for parents. Physical and occupational therapists are readily available to aid in mobilizing the patients. There is a neurosurgical resident on call 24 hours a day, 7 days a week, from home and capable of being at the hospital within 20 minutes. These factors are likely similar at most tertiary pediatric hospitals, and as our results indicate most patients could likely be monitored safely in a non-ICU setting postoperatively. The presence of an in-house neurosurgical provider (i.e., resident, nurse practitioner, etc.) at night may be beneficial when sending patients with postoperative craniotomies to a non-ICU level of care, but this remains to be proven.

Parental involvement in postoperative care of the pediatric neurosurgical patient is extremely important. In our experience, parents have not been overly concerned with the level of care to which the patient is admitted. However, this variable is difficult to assess because these conversations, if any, are not usually documented in the chart. In our experience, most parents appeared to prefer the more quiet atmosphere of the floor compared with that of the ICU. The role that parental involvement plays in the postoperative decision-making process is important and should be a factor in many pediatric neurosurgical practices.

It is important to note that we purposely excluded craniotomy for other etiologies such as vascular malformations and arachnoid cysts. It is unclear if inclusion of these patients would decrease or increase the need for ICU level of care. As such, additional studies for nontumor-related pathologies are still needed.

Our study only included 61 patients undergoing elective resection for brain tumors. Given the low total number of patients, our study is not sufficiently powered to determine clear risk factors that necessitate ICU level of care following elective resection of uncomplicated brain tumors. Further studies with larger numbers of patients are necessary to justify routine non-ICU care after elective craniotomy in children. Although adult studies with relatively large numbers of patients have been conducted, these studies are unlikely to be performed in the pediatric age group with large numbers of patients.

The cost of ICU level of care is not insubstantial. Although our study showed a relatively high transfer rate to the ICU (9.8\%), the cost in overall savings may justify this trade-off at a population-based level. In addition, most of the patients who required transfer do not appear to have suffered long-term neurological consequences as a result of the transfer.

\section{Conclusions}

Select pediatric patients undergoing elective craniotomy for brain tumor resection may benefit from ICU care in the immediate postoperative period. Other patients may be considered candidates for admission to the surgical floor for postoperative recovery. Additional studies with larger numbers of patients are needed to address which pediatric patients would benefit from ICU level of care postoperatively.

\section{References}

1. Beauregard CL, Friedman WA: Routine use of postoperative ICU care for elective craniotomy: a cost-benefit analysis. Surg Neurol 60:483-489, 2003

2. Bernstein M: Outpatient craniotomy for brain tumor: a pilot feasibility study in 46 patients. Can J Neurol Sci 28:120124, 2001

3. Boulton M, Bernstein M: Outpatient brain tumor surgery: innovation in surgical neurooncology. J Neurosurg 108:649654, 2008

4. Bui JQH, Mendis RL, van Gelder JM, Sheridan MMP, Wright KM, Jaeger M: Is postoperative intensive care unit admission a prerequisite for elective craniotomy? J Neurosurg 115:1236-1241, 2011

5. Grundy PL, Weidmann C, Bernstein M: Day-case neurosurgery for brain tumours: the early United Kingdom experience. Br J Neurosurg 22:360-367, 2008

6. Halpern NA, Bettes L, Greenstein R: Federal and nationwide intensive care units and healthcare costs: 1986-1992. Crit Care Med 22:2001-2007, 1994

7. Hanak BW, Walcott BP, Nahed BV, Muzikansky A, Mian MK, Kimberly WT, et al: Postoperative intensive care unit requirements after elective craniotomy. World Neurosurg 81:165-172, 2014

8. Hecht N, Spies C, Vajkoczy P: Routine intensive care unitlevel care after elective craniotomy: time to rethink. World Neurosurg 81:66-68, 2014

9. Phelan HA, Richter AA, Scott WW, Pruitt JH, Madden CJ, Rickert KL, et al: Does isolated traumatic subarachnoid hemorrhage merit a lower intensity level of observation than other traumatic brain injury? J Neurotrauma 31:1733-1736, 2014

10. Ratzer M, Brink O, Knudsen L, Elklit A: Posttraumatic stress in intensive care unit survivors-a prospective study. Health Psychol Behav Med 2:882-898, 2014

11. Sundararajan K, Martin M, Rajagopala S, Chapman MJ: Posttraumatic stress disorder in close Relatives of Intensive Care unit patients' Evaluation (PRICE) study. Aust Crit Care 27:183-187, 2014

12. Washington CW, Grubb RL Jr: Are routine repeat imaging and intensive care unit admission necessary in mild traumatic brain injury? J Neurosurg 116:549-557, 2012

\section{Disclosures}

The authors report no conflict of interest concerning the materials or methods used in this study or the findings specified in this paper.

\section{Author Contributions}

Conception and design: Gabel, Levy. Acquisition of data: Gabel. Analysis and interpretation of data: Gabel, Martin, Crawford. Drafting the article: Gabel, Crawford. Critically revising the article: Levy. Reviewed submitted version of manuscript: Gabel, Crawford, Levy. Approved the final version of the manuscript on behalf of all authors: Gabel. Statistical analysis: Martin. Study supervision: Levy.

\section{Correspondence}

Brandon C. Gabel, Division of Neurosurgery, University of California, San Diego, 200 W. Arbor Dr., San Diego, CA 92013. email: brandoncgabel@gmail.com. 